\title{
Calculation of $G$ Gravity Constant from the Mass and Electron Charge, and Fine Structure Constant
}

\author{
Valentino Straser \\ Department of Science, Environment and Energy, Brussels, Belgium \\ Email: valentino.straser@gmail.com,valentino.straser@europe-upkl.eu
}

How to cite this paper: Straser, V. (2021) Calculation of G Gravity Constant from the Mass and Electron Charge, and Fine Structure Constant. Journal of Modern Physics, 12, 1172-1181.

https://doi.org/10.4236/jmp.2021.128071

Received: May 2, 2021

Accepted: June 22, 2021

Published: June 25, 2021

Copyright $\odot 2021$ by author(s) and Scientific Research Publishing Inc. This work is licensed under the Creative Commons Attribution International License (CC BY 4.0).

http://creativecommons.org/licenses/by/4.0/

\begin{abstract}
This study proposes, from the theoretical point of view, the calculation of the gravitational constant $G$, made starting from the charge and the electron mass, taking the constant of the Fine Structure into examination. In the empty space, couples of virtual positron electrons dematerialize, giving virtual photon origin. They, at their time, will become electrons, positrons and so on. These transformations are made keeping the board of their "amount of movement" and when they meet the matter, these couples come, reissued depending on the field and on the matter mass. The matter is the change of the trend of their gyromagnetic movement relationship which puts under pressure. In presence of two masses, this gyromagnetic movement relationship is already partially oriented towards the other mass. From here a force is established between these two masses that give as calculated constant equal to 6.678532. This value of $G$, obtained leaving from the charge and the electron mass, is very near the experimental values estimated in these last decades regard the value of the gravitational constant of $G$.
\end{abstract}

\section{Keywords}

Electron, Positron, Gravitational Constant, String Theory, Theory of Everything, Fine Structure Constant

\section{Introduction}

This study stems from a reflection on the quantum vacuum. The vacuum field, according to the quantum concept, can have no null energy states. The question is to answer, at a theoretical level, what is the mechanism and what are the possible causes that generate energy. The real nature of the physical vacuum is a debated topic and is the basis of modern cosmology and represents a tool to un- 
derstand the foundations of physics. In this study, it is believed that the energy produced in the vacuum field comes from pairs of electrons and positrons. The electron-positron dipoles, in addition to interacting with each other producing virtual photons, affect neighboring dipoles by transferring to them "amount of motion". The contribution of this study is that, when electron and positron pairs meet particles of matter, they exert a pressure on it transferring energy that, hypothetically, is manifested as electromagnetic fluctuation. Keeping a holistic view of the physical phenomena discussed in this study and combining electron-positron electromagnetic interactions with Newtonian dynamics, it has come to the calculation of the gravitational constant $G$, resulted close to modern values calculated in the laboratory.

The Law of Universal Gravitation represents a milestone of scientific knowledge to the main thread of this study is represented by the calculation of the Gravitational Constant $G$, starting from the interaction between electron and positron. The calculation of $G$ was carried out on an atomic scale, instead of considering the large masses predicted by Newton's equation. We wonder if this constant can represent a common denominator between the behavior of particles, the structure of matter and the dynamics of celestial masses and if, at theoretical level, the constant $G$ can be considered a "mediator" between General Relativity, the forces of the Standard Model of particles and String Theory. The Law of Universal Gravitation represents a milestone of scientific knowledge to interpret celestial mechanics and a cornerstone of predictive science. In Newton's formulation and in the field equation of general relativity appears the proportionality coefficient $G$, independent of the physical location and masses used to determine it experimentally. In the last two and a half centuries, there have been experiments for the approximate calculation of $G$, from the eighteenth century to the present with increasingly refined methods and instruments [1] [2]. Recently, a team of quantum physicists from the University of Vienna and the Austrian Academy of Sciences realized for the first time in the laboratory a miniature version of the Cavendish experiment using millimeter-order masses. A result that opens new perspectives for the possible connection between gravitational and quantum physics [3] [4] [5] [6]. In this study, contrary to the techniques used in the past, such as the torsion balance or based on the principle of the pendulum, is proposed the calculation of the constant of gravitation $G$ starting from the charge and the electron mass. The calculation, expressed in theoretical form, was conducted by the Belgian physicist Fernand Léon Van Rutten, and presented posthumously, having disappeared in 2016. This is a written memoir that Belgian Physicist left to his daughter as a scientific testament. The calculation of the constant of $G$, in the Van Rutten point of view, originates from the "bricks" of the matter rather than start her big masses of the celestial bodies, according to the Newtonian concept. A universal constant being of $G$, his estimated value, leaving from the electron positron interaction with respect to the big masses, therefore represents a point of connection among the concept of "micro" and "macro" cosmos, and nominates himself as unifying element be- 
tween the fine matter and the gravity. The costing of gravitational or constant universal gravitation strength of $G$, is in fact the same one for all the bodies equipped with mass, be they as big as the stars or as little as sand grains. And, in the universe everything reduces himself to particles. Studying the world at smaller scales, on the order of the Angstrom, provides an opportunity to understand what we observe at larger scales. Of course, for each of these scales the behavior of matter is different.

The idea of a "hierarchy of universes" is not new. It, in fact, was already alive in Democritus of Abdera, understood as "scale factors", while new concepts were taken up in 1761 by J.H. Lambert and gradually developed until today, through H. Alfvén, O. Klein, D.D. Ivanenko and others [7]. Over the centuries, the need of physicists to find a formula or mechanism that brings together the four forces that interact on matter, gravity, electromagnetism, strong interaction and weak interaction, has been a common thread and an ambitious goal in the world of Physics. Recently, the study of gravity has been extended to include antimatter [8]. A holistic approach that associates physical structures, apparently different as gravity and electromagnetism, had been studied in the beginning by Michael Faraday (1849-1950) and then resumed, after about half a century by Weyl (1918) [9] and from the '20s by Albert Einstein with the "Unified Field Theory". But, after the innovations of the late 1800s and early 1900s, the search for a universal theory that encompassed the four forces that interact on matter became an insistent goal in the scientific world and among Physicists. The goal was, and is, to conceive a new theory, the "Theory of Everything", initially coined by J. Ellis (1986) [10] and pursued by Stephen Hawking [11]. Among the best-known empirical observations, the relationship between the gravitational universe and the universe of elementary particles stands out, the result of which concurs to hypothesize the existence of a similarity in a geometric and physical sense between macro universes and strong micro universes [12]. The adjective "strong" must be however contextualized in the scale physics, where the strong nuclear force that helps to keep together the matter is far superior to the other three fundamental forces: gravity, electromagnetism and weak nuclear forces. The calculation of $G$, leaving from the electron-positron analysis proposed in this study, reaches the surprising result to combine the electromagnetism with the gravity, Fine Structure with the String Theory, through a deterministic physical principle and not a mathematical formulation with the limit already highlighted by the Gödel's Theorem of Incompleteness [13]. A question, that of unifying the fundamental interactions of physics, which does not cease to arouse interest in research, also discussed in recent publications [14] [15] [16].

\section{Constants and Variables}

Etymologically and conceptually, the term "constant" ensures that some quantities remain so over time. However, in spite of their current use, the origin of "constants" is still an open question, not only in the world of Physics. Their importance cannot certainly be neglected, since different values of physical con- 
stants would radically change the knowledge of physical phenomena known up to now. In this study, the value of the Constant $G$ has been deduced using other physical constants, such as the speed of light, mass, the fine structure constant [17] [18], Plank's constant, the inverse of the fine structure constant multiplied by 0.75 , and fundamental quantities such as the electron charge. Unlike the constants used for the calculation of this work, as known validated by observation and experiment, the Gravitational Constant $G$ derived from the interaction between electron-positron, is instead theoretical in nature. Its value, however, was found to be very close to both $G$ of Newton's gravity, and to that recently calculated in the laboratories.

The variables in the calculation, however, are represented by the gyromagnetic orientation of the dipole electron-positron, which exerts pressure on matter when it intercepts it, and the distance electron-positron, which in this case must be much greater than the wavelength. From the outcome of this study, the value of the constant $G$ can be considered for the different scales of mass magnitude, from the subatomic to the cosmic universe and vice versa and, in perspective, contribute to a better definition of the value of the constant $G$ in the International System.

\section{Method}

The Method used to calculate the $G$ constant is based on the concept of the "circumstantial paradigm" [19] associated with the deductive method, i.e., guiding the logical procedure from hypothesis to conclusion. In this case, the "clue" coincides with the hypothesis that there exists a particle responsible for gravity, produced due to the interaction between electron-positron and their formation and destruction processes over time.

The procedure to realize the calculation of the constant $G$ involves the use of other physical constants and is divided into two phases. The first considers an electromagnetic interaction in the electron-positron pairs; the second phase concatenates, through the gyromagnetic ratio of dipole, electromagnetic interactions with Newtonian dynamics, from which it is possible to obtain the value of gravitational constant $G$.

\section{Discussion}

In this study, we will try to show that the gravitational constant of $G$ could be the result of a relationship between other physics constants. To explain the Universal Attraction Law, it was often assumed that a particle called graviton exists [20] [21] [22] [23]. In this study, we will show that a particle responsible for the gravity exists indeed. The space is not completely empty, it contains neutrinos, electromagnetic waves and fields, like the electromagnetic field and the gravitational field. The importing thing more, according to the cosmologists, is that the space contains most of the energy of the cosmos [24]. In this study we will show, as he says A.V. Rykov [25], that this energy could be formed by virtual couples of the 
electron and positron that arise and, they disappear, forming photons immediately [26] [27] [28]. These couples [29] could be the Strings of the last approaches to physics [30] [31] [32]. In fact, Heisemberg's uncertainty principle states that the uncertainty of energy of a particle multiplied by the uncertainty of its lifetime must be greater or equal to the Plank constant divided by $4 \pi$, because these particles to be measurable.

- Below this value, these particles are imperceptible and so-called virtual.

- The encounter of virtual particles is not new.

Already the Lamb effect [33] can only be explained by a process in which a nucleon issues a virtual meson which interacts electromagnetically with the atomic electron and is subsequently reabsorbed by the nucleus [34].

These mesons are virtual electron-positron couples or photons to high energy. We will afterwards do the following hypotheses:

1) The space is height of these virtual couples or dipoles.

2) These virtual electron and positron couples do not disappear, but are annihilated giving virtual photon which, at their time, rematerialize in electrons and positrons origin and so away, giving the appearance of a movement of the electron and positron couple which travels in the space.

3) During their short life duration, these dipoles affect the near dipoles, transmitting them part of their "Momentum". These interactions between dipoles determine, hypothetically, electromagnetic fluctuations in the empty space.

4) But above all, when they meet a matter particle, are sent to following the particle field back and therefore exercise a pressure on the matter itself.

We will say that these pairs form and destroy sinusoidally over time. Therefore, the segment which joins these two charges is crossed by a current $I$ and in complex notation, will have in Equation (1):

$$
e=I / j \omega
$$

$e$, it is the electron charge and $\omega=2 \pi v$, dove $v$ it is the oscillation frequency.

In a distant $r$ point from the dipole, the delayed potential is express as in Equation (2):

$$
A=\frac{\Gamma \mu I}{4 \pi r} e^{-\frac{j \omega r}{c}}
$$

( $\mu$, is the magnetic permeability of the medium, $\Gamma$ the distance between the electron and the positron, $c$ the light speed).

This takes, cross the classical reasoning [35], to the average flow of the vector of Poynting in direction of the dipole. If we integrate between 0 and $\pi$ we get in Equation (3):

$$
\frac{\Gamma^{2} I^{2} \int \sin ^{2} \theta \mathrm{d} \Omega}{8 \varepsilon c \lambda^{2} \int \sin \theta \mathrm{d} \Omega}=\left(\frac{\Gamma}{\lambda}\right)^{2} I^{2} 4 /(3 \pi c)
$$

( $\Omega$ is the solid angle, $\mathrm{d} \Omega=2 \pi \sin \theta \mathrm{d} \theta$, and $\lambda$ is the wavelength of the oscillation, $\varepsilon=1 / 4 \pi$ ).

This relation is only valid if the distance is large, that is for $r \gg \lambda$. 
If we substitute $P^{2}$ for its value $\mathcal{J}^{2} \omega^{2} e^{2}$ in Equation (4), we get the energy radiated during the lifetime of the dipole, that is, a time equal to $1 / v$.

$$
E=\left(\frac{\Gamma}{\lambda}\right)^{2} \frac{v 4 \pi e^{2}}{3 c} v 4 \pi e^{2}=2 h v
$$

Because, after its short life, the dipole transforms into two photons $h v$ so we get in Equation (5):

$$
\alpha=\left(\frac{\Gamma}{\lambda}\right)^{2}=\frac{3 h c}{8 \pi e^{2}}
$$

$\alpha$ is a constant, the inverse of the constant of Fine Structure, multiplied by 0.75. Max Born insists on the importance of this constant: the only one which can be formed from $e$, while $c$ and $h$ indicate a deeper relation between electrodynamics and quantum theory [36].

Now we find out what the gyromagnetic ratio $g_{0}$ of the dipole will be. Since the current in the dipole is $I=e j \omega$, the magnetic moment will be $e \omega \Gamma^{2}$ and $\lambda$ being the wavelength of the oscillation, its angular momentum will be $m \omega \lambda^{2}$ and the gyromagnetic ratio becomes $g_{0}= \pm(e / m) \alpha= \pm \gamma_{0} \alpha$.

The orientation of these $\gamma_{0}$ is determined by the direction from which they come, and we will say that, whatever their sign, these $\gamma_{0}$ exert pressure on the particles of matter they encounter.

If we take a reference axis, with respect to this axis, each of these electrified particles arrives to an angle $\mathcal{\vartheta}$, the projection of their gyromagnetic on this axis relationships will be: $\gamma \alpha \cos \vartheta$, and the variations on this axis will be in Equation (6):

$$
\mathrm{d} \gamma=\gamma \alpha \sin \vartheta \mathrm{d} \vartheta
$$

If we integrate between $\gamma_{0}$ and $\gamma$ we get: $\gamma$ in Equation (7)

$$
\log \left(\gamma_{0} / \gamma\right)=-\alpha \cos \vartheta+\text { constant }
$$

If we place the constant $=0, \gamma$ becomes in Equation (8):

$$
\gamma=\gamma_{0} e^{-\alpha \cos \vartheta}
$$

We take now in Equation (9) a particle of mass $M$ and we will say that the number of electrons positrons received with a solid angle $\mathrm{d} \Omega$ at any time is proportional to:

$$
A e^{-\alpha \cos \vartheta} \mathrm{d} \Omega
$$

A is determined by the total number of electrons positrons present.

$\gamma$ in the solid angle it will be in Equation (10):

$$
A \gamma_{0} e^{-\alpha \cos \vartheta} \mathrm{d} \Omega
$$

If we integrate the ratio of these two relations we obtain $\gamma=\gamma_{0}$.

So, in relation to a given axis, on a half-face because of $\gamma_{0}$ dipole coming from all directions, seen from this face, this mass $M$ will be subjected to a pressure that tries to push it back and its force applied will be described in Equation (11):

$$
f_{1}=M \gamma_{0} E
$$


$E$, has the dimensions of an electric field. This field may be due to the virtual electrons and positrons surrounding the mass $M$, and we will not make any other assumptions about this field.

Following the reaction action principle, for two particles at a distance $r$, on the invisible faces of the other particles, the force that tends to bring them together will be in Equation (12):

$$
f_{2}=\left(M_{1} M_{2} / r^{2}\right) \gamma_{0}^{2}
$$

On the other faces, that is, those of the other mass, $\gamma_{0}$ of the dipole coming from this other mass undergoes an average orientation perpendicular to the surface of this other mass.

Their orientation is no longer anisotropic and because, in relation to their size, these masses are very distant from each other. In first approximation, $\gamma_{0}$ is affected by the other particle, it can simply be multiplied by $\cos \vartheta$.

So, we'll have for the force that tries to repel these particles, described in Equation (13):

$$
f_{3}=\left(M_{1} M_{2} / r^{2}\right) \gamma_{0}^{2} \frac{\int \cos \theta \cdot e^{-\alpha \cos \vartheta} \mathrm{d} \Omega}{\int e^{-\alpha \cos \vartheta} \mathrm{d} \Omega}
$$

If we integrate $f_{3}$ between 0 and $\pi / 2$, in Equation (14) we obtain for average $\gamma^{2}$ of the force that moves away the two particles:

$$
\gamma_{2}=\frac{1-\alpha \cdot\left(1+\frac{1}{\alpha}\right) e^{-\alpha}}{1-e^{-\alpha}} \gamma_{0}^{2}
$$

in Equation (15) as $e^{-\alpha} \ll 1$ we can neglect it to the denominator and doing $f_{2}$ $-f_{3}$, we get the force both between the two masses, and for the gravitational constant of $G$ :

$$
G=e^{2} / m^{2}\left[1-1+\alpha\left(1+\frac{1}{\alpha}\right) e^{-\alpha}\right]
$$

If we take $e^{2} / m^{2}=2.7801987 \mathrm{E} 32$ and $\alpha=102.7770278$ we obtain the gravitational constant of $G=6.678532 \times 10^{-11} \mathrm{~m}^{3} \cdot \mathrm{kg}^{-1} \cdot \mathrm{s}^{-2}$.

This gravitational constant of $G$ is very close value the experimental values obtained in these last decades. In a conference about the value of $G$ in the 1998, the average not weighed up on 10 results is [37]: $6.6772 \times 10^{-11} \mathrm{~m}^{3} \cdot \mathrm{kg}^{-1} \cdot \mathrm{s}^{-2}$.

Jan Gundlach and Stephen Merkowitz [38] found in 2000:

$$
(6.674215 \pm 0.000092) \times 10^{-11} \mathrm{~m}^{3} \cdot \mathrm{kg}^{-1} \cdot \mathrm{s}^{-2}
$$

In Zurich the professor Schlamminger [39] found:

$$
G=(6.67404 \pm 0.00021) \times 10^{-11} \mathrm{~m}^{3} \cdot \mathrm{kg}^{-1} \cdot \mathrm{s}^{-2}
$$

More recently, Rosi and colleagues found the value [40]:

$G=6.67191(99) \times 10^{-11} \mathrm{~m}^{3} \cdot \mathrm{kg}^{-1} \cdot \mathrm{s}^{-2} \quad$ [Relative uncert.: $150 \mathrm{ppm}$ ]

While Mohr, Newell, and Taylor, proposed the value of $G$ equal to [41]: 
CODATA (2014) $G=6.67408(31) \times 10^{-11} \mathrm{~m}^{3} \cdot \mathrm{kg}^{-1} \cdot \mathrm{s}^{-2} \quad$ [Relative std. uncert.: $\left.4.7 \times 10^{-5}\right]$.

\section{Conclusions}

We conclude that the calculation of $G$ is revealed to be compatible with other experimental measures of the gravitational constant of $G$, obtained by other authors with theoretical and experimental methods.

We advanced various hypotheses with this work:

- The Strings are virtual electrons positrons dipoles.

- These dipoles pressing the matter.

- The validity to add the three quarters of the constant of the Fine Structure in the projection calculation of yon axis.

The hypothesis formulated in this study has our permission to make a first fine approach to calculate the constant of gravitation $G$ to leave only from the charge and the mass of the electron and from the Fine Structure constant. The calculation of $G$, made leaving from the mass and from the electron charge, avoids making instrumental mistakes for his determination. This study clearly shows the relationship between gravity and the electromagnetism and the Fine Structure, besides to offer, at hypothesis level, also a reflection on the antimatter and add the new pieces to the complex mosaic of "Theory of Everything".

\section{Acknowledgements}

In memory of the Belgian physicist Fernand Léon Van Rutten, special thanks to the anonymous reviewers for their insights and reflections to improve this study.

\section{Conflicts of Interest}

The author declares no conflicts of interest regarding the publication of this paper.

\section{References}

[1] Rosi, G., Cacciapuoti, L., Sorrentino, F., Menchetti, M., Prevedelli, M. and Tino, G.M. (2015) Physical Review Letters, 114, Article ID: 013001. https://doi.org/10.1103/PhysRevLett.114.013001

[2] Mohr, P.J., Taylor, B.N. and Newell, D.B. (2012) Reviews of Modern Physics, 84, 1527-1605. https://doi.org/10.1103/RevModPhys.84.1527

[3] Westphal, T., Hepach, H., Pfaff, J. and Aspelmeyer, M. (2021) Nature, 591, 225-228. https://doi.org/10.1038/s41586-021-03250-7

[4] DeWitt, B.S. (1967) Physical Review, 160, 1113-1148. https://doi.org/10.1103/PhysRev.160.1113

[5] Feynman, R.P. (1963) Acta Physica Polonica, 24, 697-722.

[6] Gionti, G. (1998) Discrete Approaches towards the Definition of Quantum Gravity. SISSA Ph.D.

[7] Weinberg, S. (1972) Gravitation and Cosmology. Principles and Applications of the General Theory of Relativity. Wiley, New York. 
[8] Jentschura, U. (2020) Physics, 2, 397-411. https://doi.org/10.3390/physics2030022

[9] Weyl, H. (1918) Gravitation und Elektrizität. Sitzungsber. Preuss. Akad. Wiss., Berlin, 465.

[10] Ellis, J. (1986) Nature, 323, 595-598. https://doi.org/10.1038/323595a0

[11] Hawking, S. (2006) The Theory of Everything: The Origin and Fate of the Universe. Special Edition, Phoenix Books, Inc., Owen Sound, Ontario.

[12] Sagnotti, A. and Sevrin, A. (2008) Rivista del Nuovo Cimento, 31, 423-455.

[13] Feferman, S. (2006) The Nature and Significance of Gödel's Incopleteness Theorem. Institute for Advanced Study, Princeton.

[14] Esposito, G. (2011) An Introduction to Quantum Gravity.

[15] Tatum, E., Seshavatharam, U. and Lakshminarayana, S. (2021) Journal of Modern Physics, 12, 739-743. https://doi.org/10.4236/jmp.2021.126047

[16] Miyashita, T. (2021) Journal of Modern Physics, 12, 859-869. https://doi.org/10.4236/jmp.2021.127054

[17] Sherbon, M. (2019) Fine-Structure Constant from Sommerfeld to Feynman. https://doi.org/10.31219/osf.io/hk8p5

[18] Alippi, A. (2020) Journal of Modern Physics, 11, 1918-1925. https://doi.org/10.4236/jmp.2020.1112120

[19] Straser, V. (2016) Journal of Geological Resource and Engineering, 5, 218-230.

[20] Arkani-Hamed, N., Georgi, H. and Schwartz, M.D. (2003) Annals of Physics, 305, 96-118. https://doi.org/10.1016/S0003-4916(03)00068-X

[21] Li, Z.F. (2013) Academic Journals, 8, 1968-1972.

[22] Gruzinov, A. (2005) New Astronomy, 10, 311-314. https://doi.org/10.1016/j.newast.2004.12.001

[23] Holstein, B.R. (2006) American Journal of Physics, 74, 1002. https://doi.org/10.1119/1.2338547

[24] Matarrese, S., Colpi, M., Gorini, V. and Moschella, U. (2014) Dark Matter and Dark Energy: A Challenge for Modern Cosmology. Springer, Berlin, 420 p.

[25] Rykov, A.V. (2001) Nature of Gravitation. 1-7. https://arxiv.org/pdf/physics/0112055.pdf

[26] Feynman, R. (1949) Physical Review, 76, 749-759. https://doi.org/10.1103/PhysRev.76.749

[27] Cabibbo, N. and Gatto, R. (1961) The Physical Review, 124, 1577-1595. https://doi.org/10.1103/PhysRev.124.1577

[28] Schwitters, R.F. and Strauch, K. (1976) Annual Review of Nuclear Science, 26, 89-149. https://doi.org/10.1146/annurev.ns.26.120176.000513

[29] Veneziano, G. (1990) Quantum Strings and the Constants of Nature. In: Zichichi, A., Ed., The Challenging Questions (Erice, 1989), Plenum Press, New York, 199-220. https://doi.org/10.1007/978-1-4615-3828-8_9

[30] Lamb, W.E. and Retherford, R.C. (1947) Physical Review, 72, 241-243. https://doi.org/10.1103/PhysRev.72.241

[31] Pasquinucci, A. and Petrini, M. (1997) Physics Letters B, 414, 288-296. https://doi.org/10.1016/S0370-2693(97)01182-9

[32] Vaudrevange, P. (2008) Grand Unification in the Heterotic Brane World.

[33] Salpeter, E.E. (1953) Physical Review, 89, 92-97. 
https://doi.org/10.1103/PhysRev.89.92

[34] Jentschura, U.D., Soffi, G., Ivanov, V.G. and Karshenboim, S.G. (1997) Physical Review, $A$, 56, 4483. https://doi.org/10.1103/PhysRevA.56.4483

[35] Bruhat, G. (1959) Cours de Physique Général a l'usage de l'enseignement supérieur scientifique et technique. Électricité, septieme édition entièrement remaniée par G. Goudet, Masson \& Cie Éditeurs, Paris, 905 p.

[36] Born, M. (1962) Atomic Physics. Seventh Edition, Blackie and Son Limited, London.

[37] Speake, C.C. and Quinn, T.J. (1999) Measurement Science and Technology, 10, 467-469. https://doi.org/10.1088/0957-0233/10/6/001

[38] Jens, H., Gundlach, S. and Merckowitz, M. (2000) Physical Review Letters, 85, 2869-2872. https://doi.org/10.1103/PhysRevLett.85.2869

[39] Schlamminger, S., Gundlach, J.H. and Newman, R.D. (2015) Physical Review, D, 91, Article ID: 121101. https://doi.org/10.1103/PhysRevD.91.121101

[40] Rosi, G., Sorrentino, F., Cacciapuoti, L., Prevedelli, M. and Tino, G.M. (2014) Nature, 510, 518-521. https://doi.org/10.1038/nature13433

[41] Mohr, P.J., Newell, D.B. and Taylor, B.N. (2016) Reviews of Modern Physics, 88, Article ID: 035009. https://doi.org/10.1103/RevModPhys.88.035009 